\title{
Null Mutation of the Arginine-Vasopressin Gene in Rats Slows Attentional Engagement and Facilitates Response Accuracy in a Lateralized Reaction Time Task
}

\author{
J David Jentsch*,', P Alexander Arguello', Luigi A Anzivino' \\ 'Department of Psychology, University of California at Los Angeles, Los Angeles, CA, USA
}

\begin{abstract}
The neurophysin vasopressin is thought to play an important role in emotional behavior and aspects of cognition in the rat, and the pathophysiology of this system has been implicated in two neurodevelopmental disorders, namely autism and schizophrenia. Genetic deficiency of vasopressin in rats, resulting from a null mutation of the vasopressin gene, causes alterations of brain development with resulting behavioral and neurochemical phenotypes in adulthood. We previously demonstrated that partial vasopressin deficiency (rats heterozygous for the null mutation) produces enhanced visuospatial attention and motor speeding. Here, the results of studies of homozygous Brattleboro rats that are fully vasopressin deficient are reported. We trained subjects to perform a lateralized reaction time task that measures visuospatial divided attention; in task conditions in which the duration of target stimuli was varied from trial to trial, homozygous Brattleboro rats showed a performance phenotype that consisted of more accurate responding for longer duration, and less accurate responding for briefer duration, target stimuli. No differences in response times were measured. Further experiments revealed that two separate processes produced this complex phenotype: a relatively slowed period of attentional engagement (resulting in compromised detection of fast onset-fast offset stimuli) that only partially masks a generally more accurate pattern of responding. These results, taken with earlier data, indicate that vasopressin plays a critical role in regulating visual attention and cognition, either directly, or via early alterations in neurodevelopment.

Neuropsychopharmacology (2003) 28, 1597-1605, advance online publication, 30 April 2003; doi: 10.1 038/sj.npp. I 300194
\end{abstract}

Keywords: attention; vasopressin; catecholamines; cognition; animal models

\section{INTRODUCTION}

Genetic vasopressin deficiency is well characterized as a model for non-nephrogenic diabetes inspidus (Grant, 2000; Jirikowski et al, 1992), but substantial evidence indicates that altered neurodevelopment and behavioral abnormalities are consequences of genetic vasopressin dysfunction as well (Boer, 1985). Much of what is known about the biological effects of vasopressin has been learned by studying the 'Brattleboro' rat, a variety of the Long-Evans strain that possesses a single nucleotide deletion in the second exon of the arginine-vasopressin gene (Grant, 2000; Schmale and Richter, 1984). The resulting frameshift causes synthesis of an altered protein that does not enter the normal secretory pathway. Rats that are homozygous (di/di)

\footnotetext{
*Correspondence: Dr JD Jentsch, Department of Psychology, University of California at Los Angeles, PO Box 951563, Los Angeles, CA 90095- |563, USA, Tel: + I 310825 8258, Fax: + I 3102065895 , E-mail: jentsch@psych.ucla.edu

Received I4 December 2002; revised 2 I February 2003; accepted 25 February 2003

Online publication: 12 March 2003 at http://www.acnp.org/citations/ Npp031203458/default.pdf
}

for the mutation have no detectable circulating vasopressin concentration, exhibit symptoms consistent with diabetes insipidus, and display a variety of behavioral, neuroanatomical and neurochemical changes, relative to homozygous wild-type rats (Boer, 1985; Grant, 2000; Insel et al, 1999; Schmale and Richter, 1984).

A number of studies have indicated that di/di Brattleboro rats exhibit deficits of Pavlovian fear conditioning (Stoehr et al, 1993), spatial working memory (Colombo et al, 1992), and prepulse inhibition (Feifel and Priebe, 2001), with heterozygous $(\mathrm{di} /+)$ rats generally showing a partial phenotype. Although these data have been interpreted as evidence that vasopressin plays an important and permissive role in the regulation of cognition, this hypothesis is qualified by our recent observation that $\mathrm{di} /+$ Brattleboro rats exhibit superior visuospatial attention and motor speeding, as assessed by a lateralized reaction time task (Jentsch, 2003). Overall, data indicate that the involvement of vasopressin, and the associated neurodevelopmental consequences of early vasopressin deficiency, is more complex than originally thought.

Vasopressin replacement in adult di/di Brattleboro rats does not restore all aspects of the behavioral, neurochem- 
ical, and neuroanatomical phenotype (Boer et al, 1995, 1984; Feenstra et al, 1990). This has led Boer and colleagues to conclude that vasopressin deficiency during the pre- and early postnatal periods contributes to the establishment of a set of brain abnormalities that persist into adulthood and that are the principle substrates of the behavioral phenotype (Boer, 1985). However, evidence for a role for vasopressin in cognition in adulthood is supported by pharmacological studies; impairments or improvements of attention, memory, and learning can be produced by vasopressin administration to normal adult subjects (Dietrich et al, 2001; Sahgal 1987, 1988; Strupp and Levitsky, 1985). Therefore, it seems that vasopressin influences the development and expression of cognitive functions via actions during the pre-, peri-, and postnatal periods.

Here, we extended upon our earlier studies of heterozygous Brattleboro rats (Jentsch, 2003) by evaluating the acquisition and performance by homozygous Brattleboro and wild-type rats of a lateralized reaction time task that measures visuospatial attention and motor initiation (Brown and Robbins, 1989; Carli et al, 1985, 1989). In the task employed, animals engage in an observing response while waiting for the presentation of a visual target stimulus at one of the two possible spatial locations; subjects report stimulus detection by making a response towards the location of the target. This task therefore taxes the monitoring aspect of divided attention, while minimizing the vigilance component. These studies were designed to evaluate whether $\mathrm{di} /+$ rats show a partial phenotype, as compared with $\mathrm{di} / \mathrm{di}$ rats, or whether the pattern of behavioral changes are qualitatively different.

\section{MATERIALS AND METHODS}

\section{Subjects}

The subjects were 60-day-old male rats (homozygous (di/di) Brattleboro or wild-type Long-Evans rats) purchased from Harlan (Indianapolis, IN) and were housed in genotypespecific groups on a $06.30-20.00 \mathrm{~h}$ light cycle; all behavioral testing was conducted during the light phase. The subjects were initially food deprived to $90 \%$ of their free-feeding weights and subsequently fed $15 \mathrm{~g}$ of rat chow/day in their home cages ( $1 \mathrm{~h}$ after testing). Water was continuously available except during the testing period; homozygous rats always had two bottles of water available to ensure that their higher demand for water was satisfied. The experimental protocols employed were consistent with the NIH 'Guide for the Care and Use of Laboratory Animals' and were approved by the Chancellor's Animal Research Committee at UCLA.

\section{Behavioral Testing Apparatus}

Standard extra-tall aluminum and Plexiglas operant chambers with a curved panel with 5 nose poke apertures on one side and a photocell-equipped pellet receptacle on the other side (Med Associates, Mt Vernon, VT) were used. The boxes were housed inside of a sound-attenuating cubicle with ambient white noise $(85 \mathrm{~dB})$ broadcast to mask external noise; the environment was illuminated with a house light (a light diffuser that was located outside of the operant chamber but within the cubicle).

\section{Pretraining}

All rats were first trained in a single session in which the house light was continuously illuminated and single pellets were delivered into an illuminated magazine on a fixed-time 20 -s schedule over a 45 -min period. At 1 day after this session, the rats were trained to make a sustained nose poke at the center aperture in three consecutive daily sessions. On the first day, the session began with illumination of the house light; a variable duration nose poke of $10,200,400$, or $600 \mathrm{~ms}$ was required in the illuminated center aperture in order to trigger a pellet to be dispensed within the head entry magazine on the back wall (the nose poke duration requirements were varied randomly from trial to trial). When the rat successfully responded for the duration of the hold period, the head entry magazine was illuminated and a pellet was dispensed. After the rat retrieved the pellet, the magazine light was extinguished, and $3 \mathrm{~s}$ later, the center aperture was illuminated to signal the initiation of another trial. The session terminated after $60 \mathrm{~min}$ passed or the rat earned 100 pellets, whichever occurred first. On the second and third days, the procedure was identical except that the rat was required to sustain $10,200,500$, or $700 \mathrm{~ms}$ nose pokes on the second day and $200,500,700$, or $1000 \mathrm{~ms}$ nose pokes on the third day.

\section{Acquisition of the Lateralized Reaction Time Task}

After being trained to make the sustained nose poke, rats began daily testing on the lateralized reaction time task (Brown and Robbins, 1989; Carli et al, 1985; Jentsch, 2003); in the first four sessions, a target stimulus of fixed duration was presented for all trials in a session (which terminated after $60 \mathrm{~min}$ or 128 trials, whichever came first). The task began with the illumination of the house light and the rats retrieving a single pellet from the magazine. The center aperture on the opposite wall was illuminated 3-s later. The rat was then required to make a sustained, variable duration nose poke $(200,500,700$, or $1000 \mathrm{~ms})$ in the center aperture. After the sustained nose poke (the 'observing response') was completed, the far left or far right aperture was illuminated for a fixed period $(30,5,2.5$, or $1 \mathrm{~s}$ on days 1,2 , 3 , or 4 of training, respectively). During target presentation, a nose poke response at that aperture resulted in a pellet being delivered at the magazine, and a 'correct' choice was scored. A limited hold period also applied on days 3 and 4; a response within $5 \mathrm{~s}$ of onset of target illumination was reinforced. At $3 \mathrm{~s}$ after the pellet was retrieved, the center aperture was illuminated to signal the onset of another trial.

When a rat responded at a location that was not that of the target during target presentation or within the limited hold period, all lights in the box were extinguished, and the rat was given a 3-s 'time-out' period in complete darkness; in this case, an 'incorrect choice' was scored. Additionally, if the rat made no response within target presentation or the limited hold period, the rat received a 3-s 'time-out' in darkness and an 'omission' was recorded. In both cases, the time-out period was immediately followed by illumination of the house light diffuser and the onset of another trial. 
An additional contingency was in place to discourage premature responses. If a rat responded to either of the possible target locations before completing the sustained nose poke (and before the target presentation), a 3-s timeout was given (as above), and an 'anticipatory response' was scored.

Dependent measures for this task included: (1) rate of both correct and incorrect choices (percentage of total trials), (2) omission rate (percentage of total trials), (3) total anticipatory responses, (4) total trials initiated, (5) mean initiation latency/trial (the average interval between illumination of the center nose poke aperture and the initiation of the observing response), (6) pellet retrieval time (the average interval between pellet delivery and head entry into the magazine), (7) correct response times (the period between target stimulus onset and a nose poke at the response location), and (8) receptacle approaches to the pellet magazine when no pellet was available. Analysis of variance (factor: genotype) was used to test for group differences for all dependent measures.

\section{Variable Target Stimulus Duration Condition}

After the acquisition period, rats were tested in sessions in which the duration of the target stimuli was varied randomly from trial to trial within the session, with the stimulus brightness being held constant. In some initial test sessions, target stimulus durations ranged between 0.5 and $4.0 \mathrm{~s}$, and a correct response within $4.0 \mathrm{~s}$ of target onset was reinforced. In subsequent test sessions, target stimulus durations ranged between 0.2 and $2.0 \mathrm{~s}$, and a correct response within $3.0 \mathrm{~s}$ of target onset was reinforced. The session ended after $60 \mathrm{~min}$ or 160 trials, whichever came first. All the other task details were identical to those described above, and the dependent measures were the same. In these sessions, rates of correct and incorrect choices, response times and omission rate were analyzed with reference to stimulus duration (repeated measures of analysis of variance). Other measures were not dependent upon stimulus duration and were analyzed independently by one-way analysis of variance.

\section{Variable Target Stimulus Brightness Condition}

Subjects were also tested in sessions in which stimulus duration was held constant $(1.0$ or $0.5 \mathrm{~s})$, while target brightness was varied, between 20 and $80 \%$ of full intensity, from trial to trial. A correct response within $3.0 \mathrm{~s}$ of target onset was reinforced. The session ended after $60 \mathrm{~min}$ or 160 trials, whichever came first. All the other task details were identical to those described above, and the dependent measures were the same. In these sessions, rates of correct and incorrect choices, response times and omission rate were analyzed with reference to stimulus brightness (repeated measures of analysis of variance). Other measures were not dependent upon stimulus brightness and were analyzed independently by one-way analysis of variance.

\section{Variable Observing Response Duration Conditions}

Animals were also tested in sessions in which the duration of the observing response required to trigger target delivery was not varied from trial to trial (as above) but held constant at either 0.2 or $1.0 \mathrm{~s}$ across all trials in the daily session. All subjects were tested in two conditions across two consecutive daily sessions; the order of these conditions was counterbalanced across all subjects to ensure that there were no main effects of order of delivery of conditions. For these conditions, we conducted $2 \times 2$ analyses of variance (factors: observing response duration and genotype) with repeated measures (target stimulus duration) where appropriate.

\section{RESULTS}

\section{Acquisition of the Lateralized Reaction Time Task}

There were few effects of genotype during the acquisition period, indicating that $\mathrm{di} / \mathrm{di}$ and wild-type rats acquired the conditional rules of the task with roughly the same efficiency (Table 1). On the first day of training on the lateralized reaction time task, when the target stimulus duration was $30 \mathrm{~s}$, di/di made fewer omissions $\left(\mathrm{F}_{(1,14)}=7.1\right.$, $p=0.02)$ and showed faster response times $\left(\mathrm{F}_{(1,14)}=9.7\right.$, $p=0.008$ ), with no other performance measures being significantly affected by genotype. There were no main effects of genotype on day 2 of training, when the stimulus duration was $5 \mathrm{~s}$. Brattleboro rats also exhibited slightly longer mean trial initiation latencies on the $2.5 \mathrm{~s}$ target condition $\left(\mathrm{F}_{(1,14)}=5.5, p=0.03\right)$. On day 4 , when the target stimulus duration was $1.0 \mathrm{~s}$, only anticipatory responses were affected by genotype $\left(\mathrm{F}_{(1,14)}=4.7, p=0.04\right)$, with $\mathrm{di} / \mathrm{di}$ rats producing more impulsive responses than did wild-type subjects. Overall, these results indicate no robust phenotypic differences in di/di and wild-type rats during acquisition.

\section{Variable Duration Task Conditions}

We subsequently tested the subjects in two consecutive test sessions (sessions 1 and 2; Figure 1) in which the durations of the target stimuli were varied randomly from trial to trial (durations were $0.5,1.0,2.0$, or $4.0 \mathrm{~s}$ ). In session 1 , correct choices were affected by target stimulus duration $\left(\mathrm{F}_{(1,14)}=47.7, p<0.0001\right)$; as predicted, all subjects were more accurate at responding towards the longer as opposed to briefer stimuli (Figure 1). A significant genotype $\times$ stimulus duration interaction $\left(\mathrm{F}_{(3,42)}=3.0, p=0.04\right)$ in the absence of a main effect of genotype $\left(\mathrm{F}_{(1,14)}=1.6\right.$, $p=0.3)$, resulted from a facilitation of choice accuracy in $\mathrm{di} / \mathrm{di}$ rats only at $2.0 \mathrm{~s}$ condition $(p=0.03)$. A genotype $\times$ stimulus duration interaction for incorrect choices $\left(\mathrm{F}_{(3,42)}=7.4, p=0.0005\right)$ resulted from higher numbers of incorrect choices in $\mathrm{di} / \mathrm{di}$ rats only at the $0.5 \mathrm{~s}$ condition $(p=0.001)$; there was no significant main effect of genotype for omissions $\left(\mathrm{F}_{(1,14)}=4.1, p=0.06\right)$. No other performance measures were affected by genotype.

Accuracy of responding in both groups was superior, overall, in session 2 compared to session 1 (this represents a practice effect), but the pattern of performance differences was unaltered (Figure 1). As in session 1, a main effect of target stimulus duration was found for correct choices $\left(\mathrm{F}_{(3,42)}=35.7, p<0.0001\right)$; all subjects showed monotonically decreasing choice accuracy with decreasing stimulus 


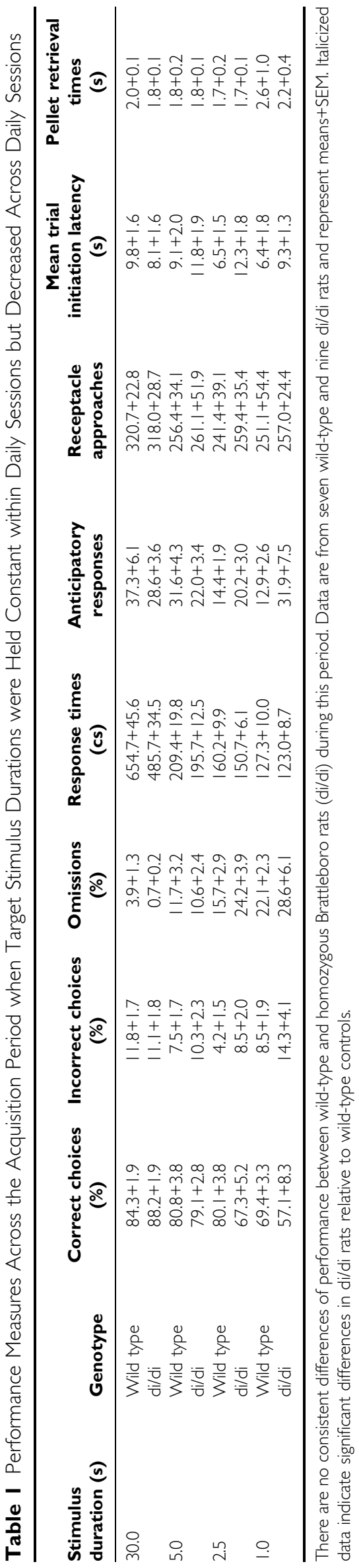

duration. There was no main effect of genotype for correct choices $\left(\mathrm{F}_{(1,14)}=0.53, \quad p=0.48\right)$; however, a genotype $\times$ stimulus duration interaction $\left(\mathrm{F}_{(3,42)}=3.9, p=0.02\right)$ pointed to relatively better performance only at the $2.0 \mathrm{~s}$ condition $(p=0.01)$. The alterations in choice accuracy were not because of altered rates of omissions in di/di rats; there was no main effect of genotype for omissions $\left(\mathrm{F}_{(1,14)}=1.9, p=0.19\right)$, nor any genotype $\times$ stimulus duration interaction $\left(\mathrm{F}_{(3,42)}=0.8, p=0.51\right)$. By contrast, there was a strong genotype $\times$ stimulus duration interaction for incorrect choices $\left(\mathrm{F}_{(3,42)}=11.8, p<0.0001\right)$, indicating that $\mathrm{di} / \mathrm{di}$ made more incorrect choices at the $0.5 \mathrm{~s}$ condition $(p=0.01)$ and fewer incorrect choices for target stimuli of $2.0-4.0 \mathrm{~s}$ duration $(p=0.004, p=0.03$, respectively). There was no main effect of genotype or genotype $\times$ stimulus duration interaction for response times. Again, neither anticipatory responses, perseverative responses, mean trial initiation latencies nor pellet retrieval latencies were affected by genotype. These results indicated that di/di rats showed a more accurate pattern of responding that was eliminated when target stimulus onset and offset was sufficiently quick. This raised the possibility that reducing target stimulus durations even further would reveal a greater impairment in di/di animals.

To address this possibility, we then tested animals in a third session in which target durations were briefer: $0.2,0.5$, 1.0 , or $2.0 \mathrm{~s}$ (see Figure 2). The results from this session confirmed our speculation. For correct choices, a strong genotype $\times$ stimulus duration interaction $\left(\mathrm{F}_{(3,42)}=10.7\right.$, $p<0.001$ ), in the absence of a main effect of genotype, was found. This interaction was because of poorer performance at the $0.2 \mathrm{~s}$ condition $(p=0.04)$ with better performance at the $2.0 \mathrm{~s}$ condition $(p=0.004)$. There was no main effect of genotype for omissions $\left(\mathrm{F}_{(1,14)}=3.6\right.$, $p=0.07)$. The duration-dependent changes in choice accuracy seemed to be driven mostly by altered rates of incorrect choices because a main effect of genotype $\left(\mathrm{F}_{(1,14)}=16.0, p=0.001\right)$ and a genotype $\times$ stimulus duration interaction $\left(\mathrm{F}_{(3,42)}=26.3, p<0.001\right)$ were found for incorrect choices. Brattleboro rats made more incorrect choices at the 0.2 and $0.5 \mathrm{~s}$ conditions $(p<0.0001$ and $p=0.0003$, respectively) and fewer incorrect choices at the 2.0 s condition $(p=0.01)$. Once again, no other performance measure, including response times, anticipatory responses, perseverative responses, mean trial initiation latencies, or pellet retrieval times, was affected by genotype.

\section{Variable Brightness Conditions}

All subjects were evaluated in two additional sessions in which the brightness of the target stimuli was varied from trial to trial $(20,40,60$, or $80 \%$ of full intensity), while target duration was held constant (1.0 s). Evaluating performance of subjects in this condition allows for an examination of the behavioral effects of manipulations of the 'detectability' but not temporal nature of target stimuli. The results from these two sessions were identical, so we only present analysis from the second of these two sessions. The data are summarized in Figure 3.

Analysis of variance revealed a main effect of genotype for both correct choices $\left(\mathrm{F}_{(1,14)}=5.3, p=0.04\right)$ and omissions $\left(\mathrm{F}_{(1,14)}=5.6, \quad p=0.04\right)$, but not incorrect choices 

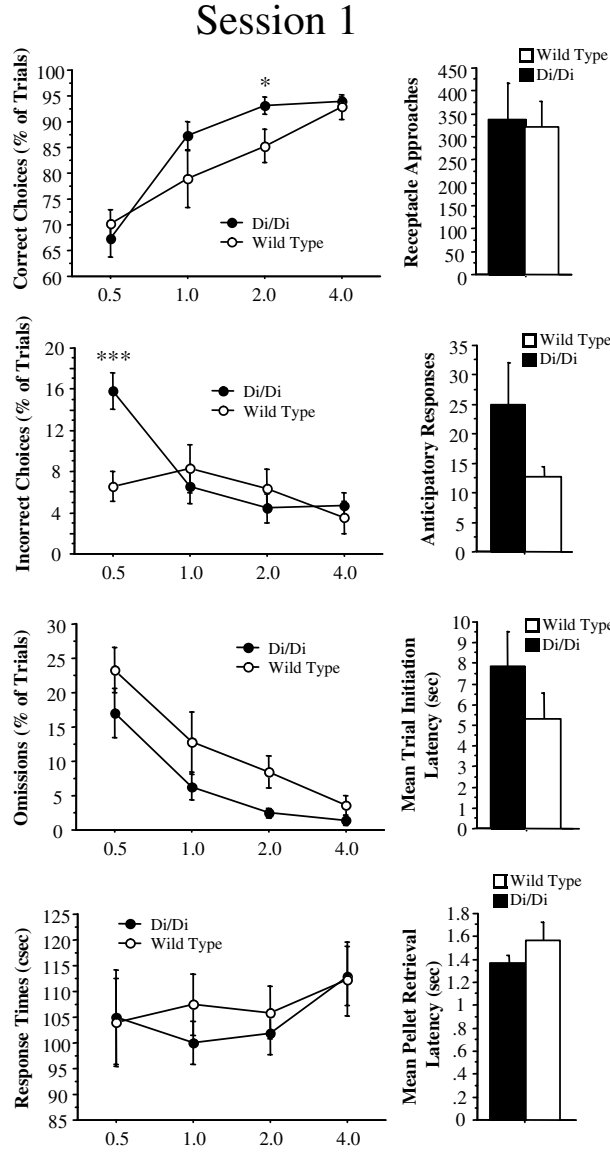

Target Stimulus Duration (sec)
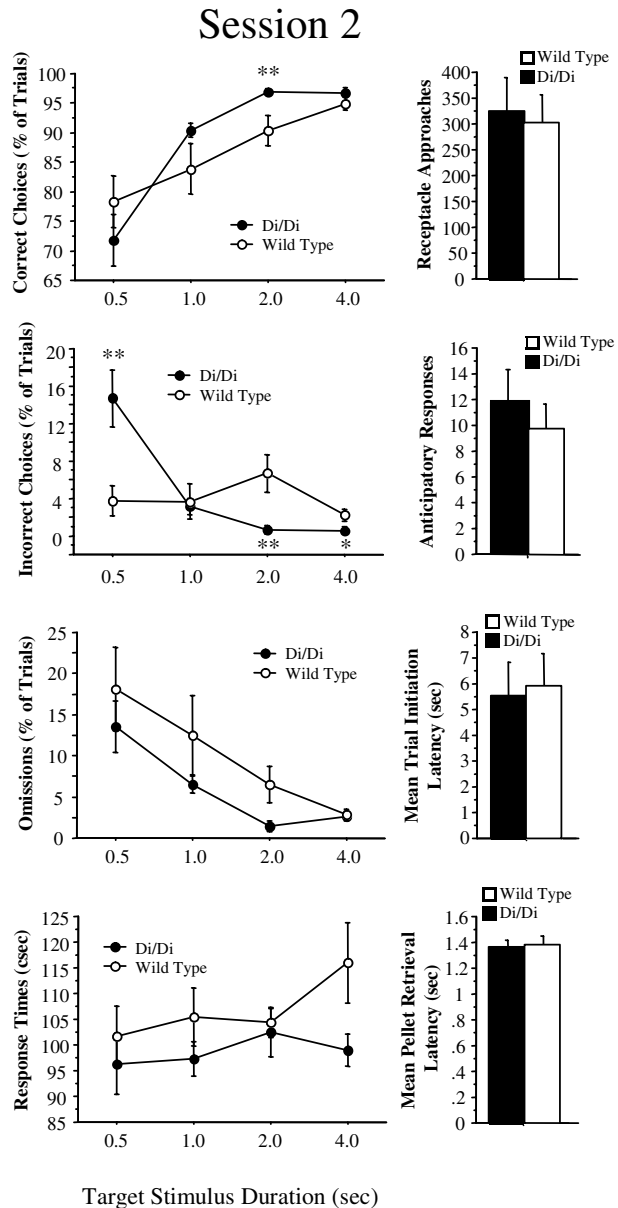

Figure I Performance of wild-type and di/di vasopressin-deficient rats in two identical daily sessions in which the duration of a visual target stimulus was varied across trials $(0.5-4.0 \mathrm{~s})$. Vasopressin-deficient rats made more correct choices for longer stimuli (2.0 s) and more incorrect choices when stimulus durations were brief $(0.5 \mathrm{~s})$. No other aspects of performance were significantly affected by genotype in either session. $* p<0.05$, $* * p<0.0$ I, *** $p<0.00$ I vs wild-type performance. $N=7$ wild-type and $N=9$ di/di rats.

$\left(\mathrm{F}_{(1,14)}=0.17, p=0.69\right)$ or response times $\left(\mathrm{F}_{(1,14)}=0.14\right.$, $p=0.71)$. These main effects were driven by a more accurate performance and fewer omissions in di/di relative to wild-type rats (roughly consistent with a slightly more accurate performance in di/di rats under the $1.0 \mathrm{~s}$ condition in the variable duration task). Neither of the main effects interacted with stimulus brightness, indicating stimulus salience did not dramatically affect the choice accuracy facilitation or decreased omissions exhibited by di/di rats under these conditions. As in the variable duration test sessions, anticipatory responses, perseverative responses, mean initiation latencies, and pellet retrieval times were unaffected by genotype.

\section{Variable Observing Response Duration Periods}

We hypothesized that the unique pattern of performance exhibited by di/di rats may be because of an interaction of two separate phenomena. We theorized that the relatively poorer ability of Brattleboro rats to detect the briefest stimuli may be because of a compromised speed of engaging attention when a trial is initiated. That is to say, when the duration of a stimulus is brief, its offset may occur more quickly than the ability of the Brattleboro rats to engage attention to the two locations that they must monitor. However, the performance deficit produced by the slowing of attentional engagement would be mitigated when the stimuli are longer in duration. Indeed, much like heterozygous Brattleboro animals (Jentsch, 2003), di/di rats show superior detection of longer duration target stimuli, possibly suggesting that they exhibit a more efficient response strategy or superior visual attention.

To address the first hypothesis, we tested rats in sessions in which the duration of the observing response which triggered target delivery was not varied randomly from trial to trial (as above), but fixed at either 0.2 or $1.0 \mathrm{~s}$ across all trials (all subjects were tested on both conditions in a counterbalanced design). If $\mathrm{di} / \mathrm{di}$ rats exhibit relatively slower engagement times, we hypothesized that the requirement of a $0.2 \mathrm{~s}$ observing response period should exacerbate their deficit (by globally reducing the time between trial initiation and target onset), while requiring a $1.0 \mathrm{~s}$ observing response period should alleviate their deficit (by providing the subjects more time to engage their attention to the two possible target locations).

Considering correct choices, two-way analysis of variance revealed no main effects of either genotype $\left(\mathrm{F}_{(1,28)}=0.4\right.$, $p=0.56)$ or observing response duration $\left(\mathrm{F}_{(1,28)}=3.3\right.$, 

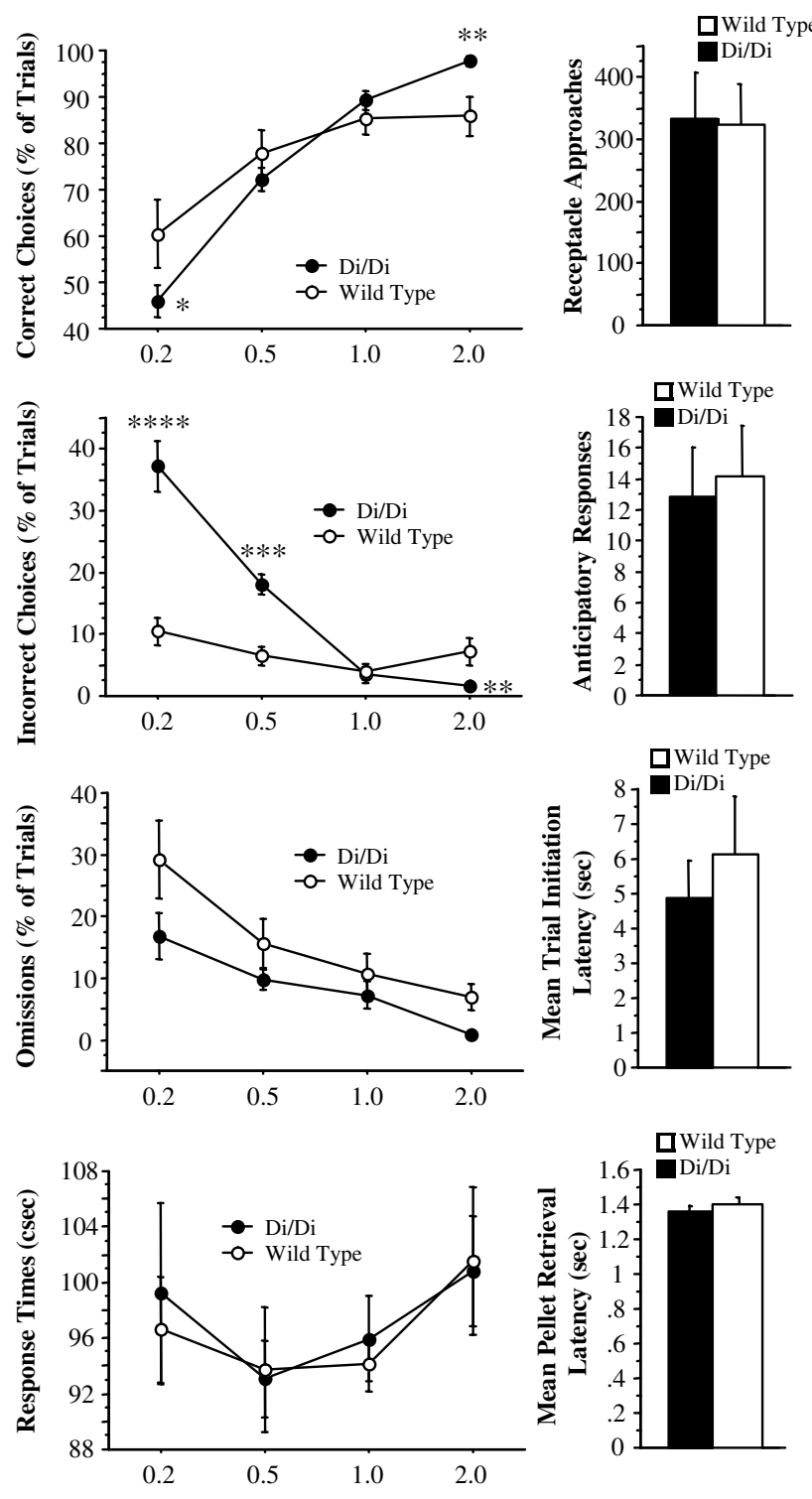

Target Stimulus Duration ( $\mathrm{sec}$ )

Figure 2 Performance of wild-type and di/di vasopressin-deficient rats in a session in which the duration of a visual target stimulus was varied across trials $(0.2-2.0 \mathrm{~s})$. Di/di subjects were impaired at responding correctly to very brief stimuli $(0.2 \mathrm{~s})$, but showed facilitated detection of longer stimuli $(2.0 \mathrm{~s})$. No other aspects of performance were significantly affected by genotype in either session. ${ }^{*} p<0.05$, $* * * 0.01$, $* * * * 0.001$ vs wild-type performance. $N=7$ wild-type and $N=9$ di/di rats.

$p=0.08)$ but a significant genotype $\times$ observing response duration interaction $\left(\mathrm{F}_{(1,28)}=5.6, p=0.02\right)$. Figure 4 shows that wild-type animals are not sensitive to the observing response duration; control animals showed equivalent numbers of correct choices, irrespective of the required duration. By contrast, di/di subjects were affected by the observing response duration; post hoc tests revealed that Brattleboro rats showed a higher percentage of correct choices, overall, than wild-type rats when the observing response duration was $1.0 \mathrm{~s}(p=0.006)$, but this difference was reversed when the observing response was $0.2 \mathrm{~s}$.

The genotype $\times$ observing response duration interaction for correct choices was mostly driven by a parallel change in
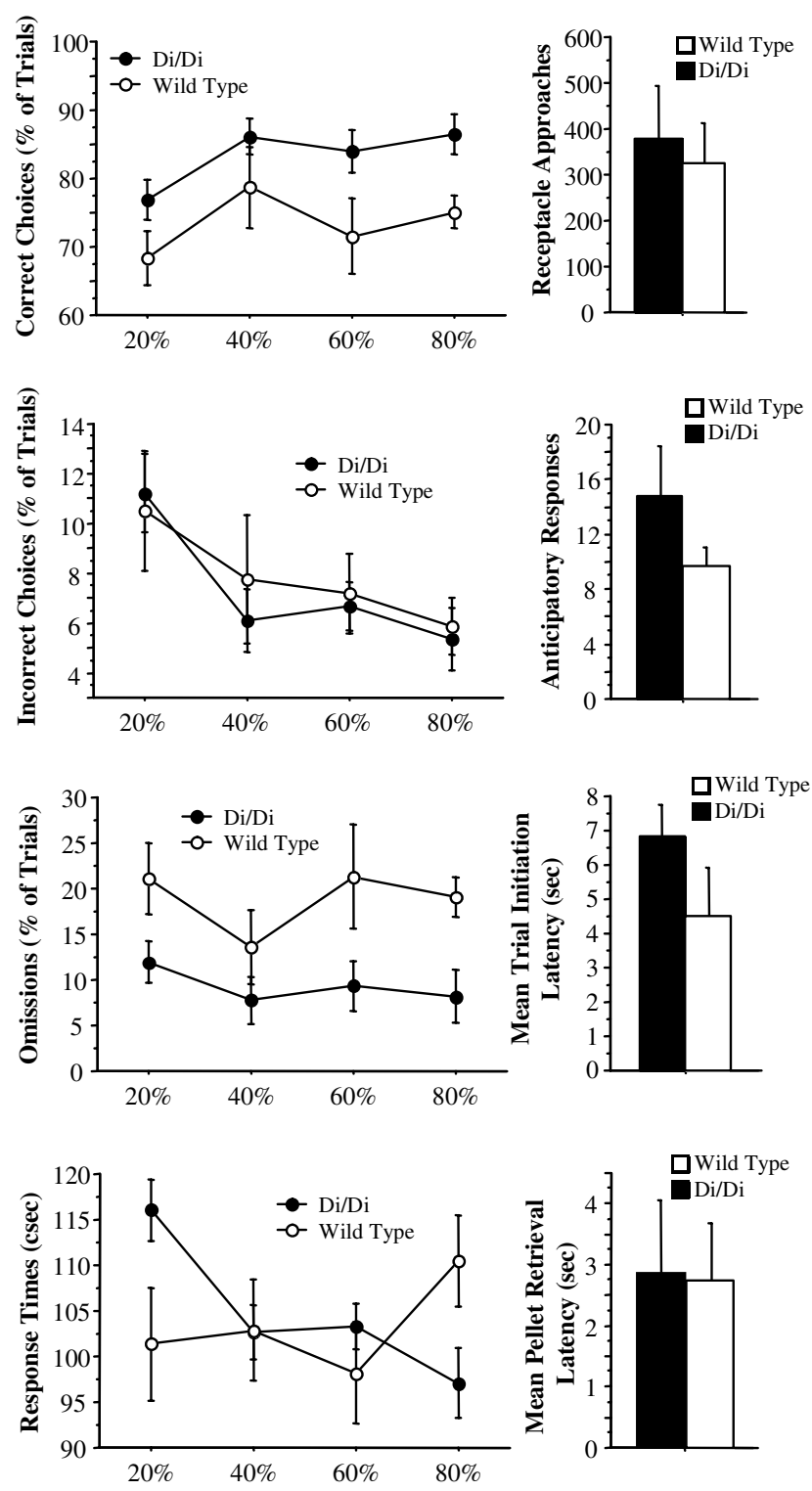

Target Stimulus Brightness (\% of Full Intensity)

Figure 3 Performance of wild-type and di/di vasopressin-deficient rats in a session in which the brightness of a visual target stimulus was varied across trials (20-80\% of full intensity; duration set at $1.0 \mathrm{~s}$ ). Di/di rats showed a generally more accurate pattern of responding that was not affected by stimulus brightness. No other aspects of performance were significantly affected by genotype in either session. $N=7$ wild-type and $N=9 \mathrm{di} / \mathrm{di}$ rats.

incorrect choices, rather than omissions. Analysis of variance detected the main effects of genotype $\left(\mathrm{F}_{(1,28)}=10.8, p=0.003\right)$ and observing response duration $\left(\mathrm{F}_{(1,28)}=6.4, p=0.02\right)$ and a genotype $\times$ observing response duration interaction $\left(\mathrm{F}_{(1,28)}=10.7, p=0.003\right)$ for incorrect choices. As with correct choices, incorrect choices in wildtype rats were unaffected by observing response duration $(p=0.3)$; however, di/di rats made significantly more incorrect choices when the observing response duration was $0.2 \mathrm{~s}$ as opposed to $1.0 \mathrm{~s}(p=0.001)$.

Considering the response times, there was no significant main effect of genotype $\left(\mathrm{F}_{(1,28)}=1.8, p=0.19\right)$, but there 

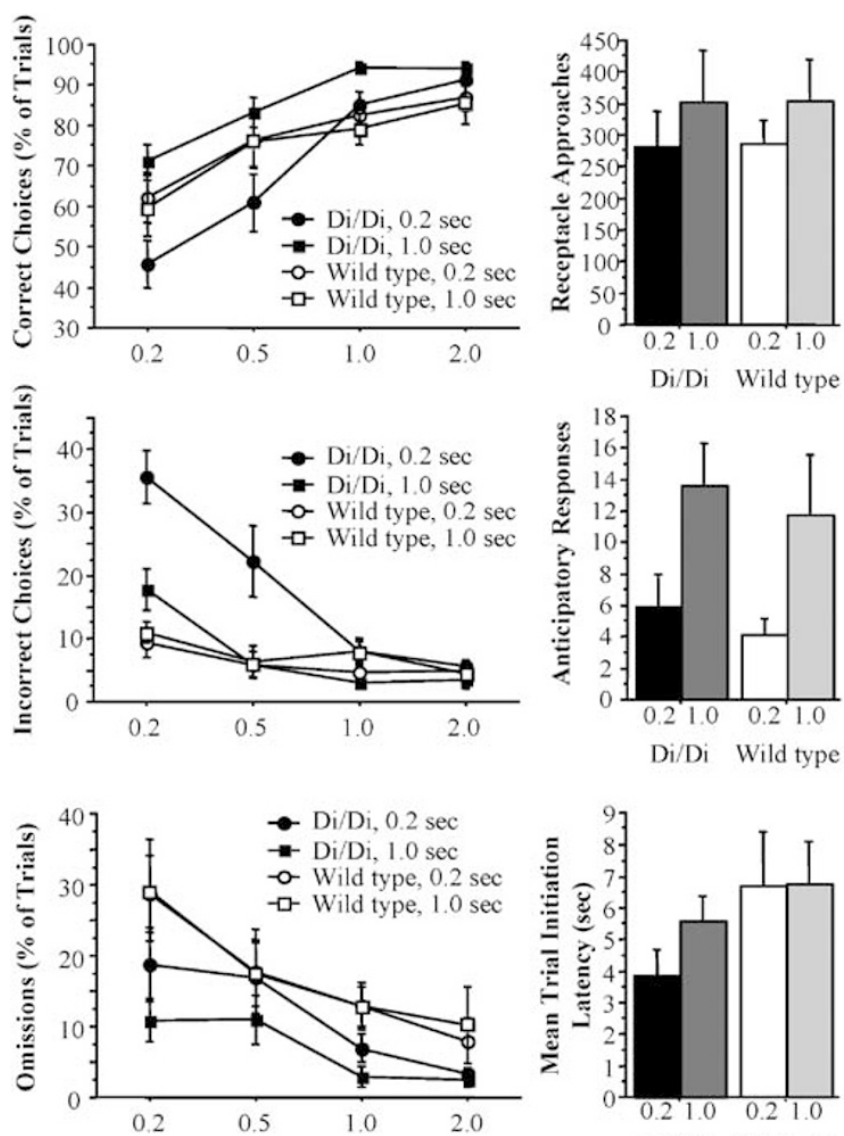

$\mathrm{Di} / \mathrm{Di}$ Wild type
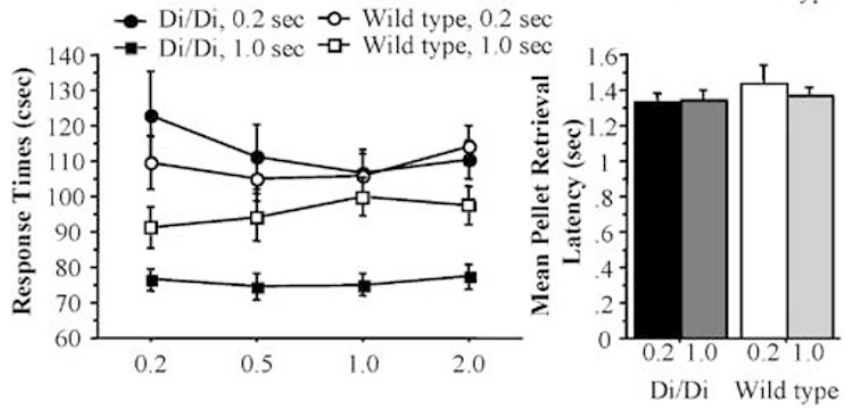

Target Stimulus Duration (sec)

Figure 4 Performance of wild-type and di/di vasopressin-deficient rats in two sessions in which the duration of the observing response required to trigger target delivery was varied across sessions but not across trials (fixed at either 0.2 or $1.0 \mathrm{~s}$ in the sessions). Di/di rats were impaired at detecting brief stimuli when the observing response period was brief $(0.2 \mathrm{~s})$ but showed facilitated performance when this period was extended $(1.0 \mathrm{~s})$. $N=7$ wild-type and $N=9$ di/di rats.

was a significant main effect of observing response duration $\left(\mathrm{F}_{(1,28)}=18.2, p=0.0002\right)$, and the genotype $\times$ observing response duration interaction nearly reached significance $\left(\mathrm{F}_{(1,28)}=4.2, p=0.05\right)$. Overall, the main effect of observing response duration was produced by faster responding when the $1.0 \mathrm{~s}$ fixation duration was in place $v s$ the $0.2 \mathrm{~s}$ duration fixation response; this difference was highly significant in di/di rats $(p<0.0001)$ and wild-type rats $(p=0.0039)$.

Anticipatory responses were elevated in both wild-type and $\mathrm{di} / \mathrm{di}$ rats when the observing response duration was 1.0 $v s 0.2 \mathrm{~s}$, as revealed by a significant main effect of observing response duration $\left(\mathrm{F}_{(1,28)}=8.4, p=0.007\right)$ with no main effect of genotype $\left(\mathrm{F}_{(1,28)}=0.5, p=0.5\right)$ and no genotype $\times$ observing response duration interaction $\left(\mathrm{F}_{(1,28)}=0.003, p=0.9\right)$. No other measures were affected by genotype or observing response duration.

\section{DISCUSSION}

Rats that are homozygous for a null mutation of the arginine-vasopressin gene exhibit robust and stable alterations of performance as assessed with a lateralized reaction time task. In this test, animals engage in an observing response (a nose poke into an aperture, the duration of which is randomly varied from trial to trial) to initiate a visual target stimulus at one of the two possible lateral locations. Subjects then respond to the correct location to obtain reinforcement. Vasopressin-deficient (di/di) rats exhibited more accurate responding to relatively longer duration target stimuli and less accurate responding to relatively briefer duration stimuli in the task. Generally, di/ di rats did not show consistently higher rates of impulsive responses or speeded response times, unlike rats that are heterozygous for the mutation (Jentsch, 2003). We hypothesize that these data indicate that $\mathrm{di} / \mathrm{di}$ rats have a generally more accurate pattern of responding to visual stimuli coupled to a slowing of attentional engagement.

Subsequent studies in which the duration of the required observing response was held constant (rather than varied randomly from trial to trial) confirmed the hypothesis that the decrement of detection of the briefest stimuli in di/di rats most likely reflects a slowing of attentional engagement. In theory, when an animal initiates the observing response, it must divide and engage attention to both possible lateral locations in order to monitor those positions for target presentation. Wild-type rats appear to be able to engage attention quite rapidly: their accuracy of responding towards visual targets was identical whether the observing response duration was 0.2 or $1.0 \mathrm{~s}$, indicating that they are capable of initiating monitoring of the target locations within that time period. These data also generally validate the usage of a variable duration observing response in wildtype rats, as altering this period does not affect choice accuracy in normal subjects.

By contrast, decreasing the duration of the observing response period (and thereby reducing the time available to engage attention prior to target delivery) exacerbated performance deficits of the di/di rats at the briefest target durations, while increasing the duration of the observing response period had the opposite effect. These results indicate that di/di rats have a relatively slowed speed of attentional engagement but that, once monitoring has been successfully initiated, they exhibit a more accurate pattern of responding.

\section{COMPARISON WITH HETEROZYGOUS BRATTLE- BORO RATS}

A comparison of performance of heterozygous and homozygous animals may serve to determine whether the Brattleboro phenotype is 'dose responsive', that is, whether a progressively greater phenotype is associated with a greater extent of vasopressin deficiency. Both heterozygous 
and homozygous animals show signs of facilitated choice accuracy under some conditions in the lateralized reaction time task. Heterozygous animals showed superior choice accuracy only when the target stimuli were brief (e.g., $0.5 \mathrm{~s}$ ), indicating that their phenotype was evident solely under the most difficult trial conditions (Jentsch, 2003). Homozygous animals show a more general pattern of choice accuracy facilitation, including generally facilitated choice accuracy when the fixation response duration is long. By contrast, heterozygous Brattleboro rats show little evidence of the slowed attentional engagement that homozygotes exhibit. Therefore, this latter phenotype may be specific for animals that are completely vasopressin deficient.

The effects of varying degrees of vasopressin deficiency on visual attention are also in concert with previous work demonstrating that systemic administration of vasopressin analogues (that act as vasopressin receptor agonists) impairs visual attention in the rat (Sahgal, 1988). Taken together, these results would seem to indicate that greater vasopressinergic tone exerts a generally negative effect on the accuracy of response selection in attentional tasks; clearly further work also needs to be undertaken to determine whether vasopressin agonists/antagonists affect attentional engagement.

\section{INTERACTION WITH DEVELOPMENT?}

Some of the neuroanatomical and neurochemical changes found in homozygous Brattleboro rats are not restored by vasopressin administration during adulthood, leading to the hypothesis that a portion of the di/di phenotype results from vasopressin deficiency during neurodevelopment (Boer, 1985). Regarding the attentional phenotype we have reported in Brattleboro rats, the relevance of the ontogenetic specificity of these effects is unknown. If vasopressin deficiency in adulthood alone contributes to these effects, we hypothesize that centrally acting vasopressin receptor antagonists may have some beneficial effects on attentional, and possibly other cognitive, functions. If early vasopressin deficiency is critical, there may be no benefit to these antagonists given in adulthood alone. The recent development of highly selective, nonpeptide vasopressin receptor antagonists (Serradeil-Le Gal et al, 2002) should allow for this issue to be addressed directly.

\section{NEURAL CIRCUITRY}

The neural mechanisms by which exogenous vasopressin administration or genetic vasopressin deficiency affect attention are unclear. The divided and/or sustained aspects of visual attention are thought to depend upon dorsal frontal cortical regions and the inputs of these structures to the striatum (Buchsbaum et al, 1990; Burk and Mair, 2001; Christakou et al, 2001; Gill et al, 2000; Muir et al, 1996; Passetti et al, 2000), while the initiation and selection of appropriate responses depend upon striatal circuitry and its modulation by dopamine (Brown and Robbins, 1989; Carli et al, 1985, 1989). In rodents and primates, there is no vasopressin input to these forebrain regions (Wang et al, 1997; Young et al, 1999), suggesting that the effects of vasopressin are indirectly mediated by other neuronal systems, such as monoaminergic and/or cholinergic nuclei (Boer et al, 1995; Caffe et al, 1985; Feenstra et al, 1990). However, it should be noted that vasopressin receptors are present in frontal cortical regions (Young et al, 1999); vasopressin may therefore reach these receptors after nonsynaptic, hormonal release, and in this way, a deficiency of transmission onto these receptors might mediate the alterations of attention reported here.

\section{CONCLUSIONS}

These data indicate that complete vasopressin deficiency results in relatively slowed attentional engagement and (under some conditions) greater choice accuracy, as assessed by a lateralized reaction time task. In principle, these data raise the possibility that antagonizing central vasopressin transmission may serve to augment visual attention. With this in mind, investigations of the effects of newly developed vasopressin receptor antagonists should include evaluations of cognitive and attentional functions.

\section{REFERENCES}

Boer GJ (1985). Vasopressin and brain development: studies using the Brattleboro rat. Peptides 6(Suppl 1): 49-62.

Boer GJ, Feenstra MG, Botterblom MJ, Korse V, Te Riele P (1995). Early postnatal appearance of enhanced noradrenaline content in the brain of vasopressin-deficient Brattleboro rat; normal adrenoceptor densities and aberrant influences of vasopressin treatment. Int J Dev Neurosci 13: 63-74.

Boer GJ, Kragten R, Kruisbrink J, Swaab DF (1984). Vasopressin fails to restore postnatally the stunted brain development in the Brattleboro rat, but affects water metabolism permanently. Neurobehav Toxicol Teratol 6: 103-109.

Brown VJ, Robbins TW (1989). Deficits in response space following unilateral striatal dopamine depletion in the rat. $J$ Neurosci 9: 983-989.

Buchsbaum MS, Nuechterlein KH, Haier RJ, Wu J, Sicotte N, Hazlett E, Asarnow R, Potkin S, Guich S (1990). Glucose metabolic rate in normals and schizophrenics during the Continuous Performance Test assessed by positron emission tomography. Br J Psychiatry 156: 216-227.

Burk JA, Mair RG (2001). Effects of intralaminar thalamic lesions on sensory attention and motor intention in the rat: a comparison with lesions involving frontal cortex and hippocampus. Behav Brain Res 123: 49-63.

Caffe AR, van Leeuwen FW, Buijs RM, de Vries GJ, Geffard M (1985). Coexistence of vasopressin, neurophysin and noradrenaline immunoreactivity in medium-sized cells of the locus coeruleus and subcoeruleus in the rat. Brain Res 338: 160-164.

Carli M, Evenden JL, Robbins TW (1985). Depletion of unilateral striatal dopamine impairs initiation of contralateral actions and not sensory attention. Nature 313: 679-682.

Carli M, Jones GH, Robbins TW (1989). Effects of unilateral dorsal and ventral striatal dopamine depletion on visual neglect in the rat: a neural and behavioural analysis. Neuroscience 29: 309-327.

Christakou A, Robbins TW, Everitt BJ (2001). Functional disconnection of a prefrontal cortical-dorsal striatal system disrupts choice reaction time performance: implications for attentional function. Behav Neurosci 115: 812-825.

Colombo G, Hansen C, Hoffman PL, Grant KA (1992). Decreased performance in a delayed alternation task by rats genetically deficient in vasopressin. Physiol Behav 52: 827-830. 
Dietrich A, Taylor JT, Passmore CE (2001). AVP (4-8) improves concept learning in PFC-damaged but not hippocampaldamaged rats. Brain Res 919: 41-47.

Feenstra MG, Snijdewint FG, Van Galen H, Boer GJ (1990). Widespread alterations in central noradrenaline, dopamine, and serotonin systems in the Brattleboro rat not related to the local absence of vasopressin. Neurochem Res 15: 283-288.

Feifel D, Priebe K (2001). Vasopressin-deficient rats exhibit sensorimotor gating deficits that are reversed by subchronic haloperidol. Biol Psychiatry 50: 425-433.

Gill TM, Sarter M, Givens B (2000). Sustained visual attention performance-associated prefrontal neuronal activity: evidence for cholinergic modulation. J Neurosci 20: 4745-4757.

Grant FD (2000). Genetic models of vasopressin deficiency. Exp Physiol 85(Spec no): 203S-209S.

Insel TR, O'Brien DJ, Leckman JF (1999). Oxytocin, vasopressin, and autism: is there a connection? Biol Psychiatry 45: 145-157.

Jentsch JD (2003). Genetic vasopressin deficiency facilitates performance of a lateralized reaction time task: altered attention and motor processes. J Neurosci 23: 1066-1071.

Jirikowski GF, Sanna PP, Maciejewski-Lenoir D, Bloom FE (1992). Reversal of diabetes insipidus in Brattleboro rats: intrahypothalamic injection of vasopressin mRNA. Science 255: 996-998.

Muir JL, Everitt BJ, Robbins TW (1996). The cerebral cortex of the rat and visual attentional function: dissociable effects of mediofrontal, cingulate, anterior dorsolateral, and parietal cortex lesions on a five-choice serial reaction time task. Cereb Cortex 6: $470-481$.
Passetti F, Humby T, Everitt BJ, Robbins TW (2000). Mixed attentional and executive deficits in medial frontal cortex lesioned rats. Psychobiology 28: 261-271.

Sahgal A (1987). Contrasting effects of vasopressin, desglycinamide-vasopressin and amphetamine on a delayed matching to position task in rats. Psychopharmacology (Berl) 93: 243-249.

Sahgal A (1988). Vasopressin and amphetamine, but not desglycinamide vasopressin, impair positively reinforced visual attention performance in rats. Behav Brain Res 29: 35-42.

Schmale H, Richter D (1984). Single base deletion in the vasopressin gene is the cause of diabetes insipidus in Brattleboro rats. Nature 308: 705-709.

Serradeil-Le Gal C, Wagnon J, Valette G, Garcia G, Pascal M, Maffrand JP, Le Fur G (2002). Nonpeptide vasopressin receptor antagonists: development of selective and orally active V1a, V2 and V1b receptor ligands. Prog Brain Res 139: 197-210.

Stoehr JD, Cheng SW, North WG (1993). Homozygous Brattleboro rats display attenuated conditioned freezing responses. Neurosci Lett 153: 103-106.

Strupp BJ, Levitsky DA (1985). A mnemonic role for vasopressin: the evidence for and against. Neurosci Biobehav Rev 9: 399-411.

Wang Z, Toloczko D, Young LJ, Moody K, Newman JD, Insel TR (1997). Vasopressin in the forebrain of common marmosets (Callithrix jacchus): studies with in situ hybridization, immunocytochemistry and receptor autoradiography. Brain Res 768: 147-156.

Young LJ, Toloczko D, Insel TR (1999). Localization of vasopressin (V1a) receptor binding and mRNA in the rhesus monkey brain. $J$ Neuroendocrinol 11: 291-297. 